\title{
El problema de la función en evo-devo*
}

\author{
The problem of function in evo-devo
}

\author{
LAURA NUÑO DE LA ROSA \\ Konrad Lorenz Institute for Evolution and Cognition Research (Austria)
}

\section{RESUMEN}

Si bien el problema de la forma ha sido el explanandum clásico de la evo-devo, frente al énfasis neodarwinista en la adaptación, en los últimos años un número creciente de autores ha reivindicado la incorporación del problema de la función en la perspectiva ontogenética de la evolución. Tras abordar los distintos modos en los que la evo-devo actual investiga la dimensión funcional de la evolución, defendemos que la distancia teórica entre la Síntesis Moderna y la 'Síntesis Extendida' no puede interpretarse en términos de la dialéctica Forma/Función. Al contrario: la 'evo-devo funcional' implica una radical reconceptuación de las funciones y plantea formas de síntesis alternativas con la teoría sintética.

\section{PALABRAS CLAVE}

EVO-DEVO, SÍNTESIS MODERNA, FORMA ORGÁNICA, FUNCIÓN BIOLÓGICA

\section{ABSTRACT}

Although the problem of form has been the classical explanandum of evo-devo vs. the Darwinist emphasis on adaptation, in the last years the problem of function has joined evo-devo's agenda. After examining the various ways in which contemporary evo-devo deals with the functional dimension of evolution, I will argue that the theoretical distance between the Modern Synthesis and evo-devo cannot be interpreted as a dialectics between Form and Function. On the contrary: the new 'functional evo-devo' implies a radical reconceptualisation of the concept of function and opens new ways of synthesis with the Modern Synthesis.

* Este trabajo recoge parte del contenido del capítulo 3 de mi tesis doctoral (Nuño de La Rosa 2012). 


\author{
KEYWORDS \\ EVO-DEVO, MODERN SYNTHESIS, ORGANISMAL FORM, BIOLOGICAL \\ FUNCTION
}

\title{
I. INTRODUCCIÓN
}

FRENTE AL ADAPTACIONISMO NEODARWINISTA ${ }^{1}$, el problema de la forma ha sido el principal explanadum de la biología evolutiva del desarrollo. Rebelándose contra la exclusión de la morfología y la embriología de la Síntesis Moderna (Love y Raff 2003), la evo-devo se propuso investigar los mecanismos ontogenéticos que constriñen la generación de las formas al margen de su valor adaptativo. En este nuevo marco teórico, la lógica del morfoespacio revelada por las homologías, las novedades morfológicas o los planes corporales debía explicarse atendiendo a la lógica del desarrollo (Alberch 1980).

El ‘espíritu morfológico’ de la evo-devo tiene, no obstante, raíces más profundas que la mera reactividad ante el 'panseleccionismo' neodarwinista. Tanto la morfología evolucionista como la biología del desarrollo, las dos grandes disciplinas que convergieron en la fundación de la evo-devo, se habían dedicado fundamentalmente al estudio de la forma pura. Por un lado, la embriología se había centrado históricamente en comprender la generación de las estructuras y no de las funciones, una tendencia que continuó a lo largo del siglo XX, tanto en la investigación genética de la morfogénesis (Carroll 2005, p. 11) como en el estudio de los mecanismos responsables de la formación de patrones (Salazar-Ciudad et al. 2003). Cuando en los ochenta la evolución comienza a contemplarse desde la perspectiva del desarrollo, esta histórica desconexión entre la fisiología y la embriología contribuyó a reforzar la investigación de las constricciones del desarrollo como constricciones sobre la forma (Amundson 1994). Por otro lado, el acento de la evo-devo en la 'forma pura', divorciada de la función y del entorno ecológico, se remonta a la morfología evolucionista decimonónica. La elegante solución que ofreciera el Origen a los problemas de la morfología permitió que la morfología pura se convirtiera rápidamente al evolucionismo. La relativa separación entre las teorías del origen común y la selección natural permitió que la anatomía y la embriología comparadas conservaran la definición de sus conceptos básicos y la metodología para el

1 Como denunciaran célebremente Gould y Lewontin (1979), la concepción molecular de la variación como arbitraria e isotrópica que asumiera la teoría sintética sugería que la selección producía soluciones óptimas en un sentido ingenieril, optimizando cada rasgo para realizar las funciones que conferirían una aptitud máxima al organismo. 
establecimiento de las relaciones morfológicas. Puesto que la unidad estructural y la diversidad adaptativa respondían a causalidades independientes (a saber: la comunidad de descendencia y la selección natural), la morfología evolucionista pudo limitarse a caracterizar las correspondencias morfológicas, ignorando los mecanismos adaptativos responsables de las variaciones específicas.

Desde la década de los setenta, la evo-devo se ha propuesto recuperar el programa de investigación de la morfología evolucionista desde una perspectiva mecanicista. El problema de la forma vuelve a aparecer, así, como principal explanandum de la biología evolucionista, pero en lugar de utilizar las relaciones morfológicas como evidencia para reconstruir relaciones filogenéticas, la evodevo aspira a comprender las causas ontogenéticas de la evolución morfológica. Este énfasis en la forma ha llevado a interpretar el conflicto entre la evo-devo y la Síntesis Moderna en el marco de la dialéctica conceptual entre forma y función, una disyuntiva que ciertos autores han utilizado también para reconstruir la historia de la biología. En este sentido, Amundson (1998) ha reivindicado la dialéctica Forma/Función para conceptuar las 'cosmovisiones' de la biología que se enfrentaron tanto en el siglo XIX (la anatomía fisiológica de Cuvier vs. la morfología pura de Geoffroy) como en la biología contemporánea (el adaptacionismo darwinista vs. el estructuralismo de la evo-devo).

Sin duda, las diferencias entre la evo-devo y la Síntesis Moderna pueden interpretarse (en parte) en el marco del conflicto entre forma y función. Sin embargo, como argumentaremos a lo largo de este artículo, existen dos grandes razones que nos obligan a matizar radicalmente esta lectura. En primer lugar, si en lugar de limitarnos a contraponer los programas de investigación de la teoría sintética y la evo-devo, atendemos a la heterogeneidad de la propia evo-devo, nos encontramos con que la dialéctica Forma/Función aparece como una tensión interna a la nueva síntesis entre evolución y desarrollo. Y es que si bien la mayor parte de los biólogos evolutivos del desarrollo ha enfatizado la perspectiva estructuralista de la evolución, ciertos autores han reclamado también la investigación de las funciones desde una perspectiva ontogenética, apostando por una "evo-devo funcional" (Breuker et al. 2006). En segundo lugar, hemos de tener en cuenta que el concepto de función no es unívoco en la biología evolutiva: si en el darwinismo la función se identifica con la adaptación ecológica y resulta de una dinámica externa a los propios organismos, el apego a los principios constructivos que rigen la organización de los seres vivos ha orientado la investigación morfológica de las funciones en una dirección muy distinta. En este sentido, creemos que ni el pasado ni el presente de la biología pueden analizarse en torno a la dicotomía Forma/Función o estructuralismo/ funcionalismo, pues las nociones de función que se han manejado en la tradición morfológica, incluyendo a la evo-devo, son muy distintas del concepto 
adaptacionista articulado en la tradición darwinista (Etxeberria y Nuño de la Rosa 2009).

Dedicamos este ensayo a examinar el problema de la función en la evo-devo contemporánea, teniendo en cuenta la raigambre histórica de esta problemática. Con tal fin, distinguimos tres modos en los que la evo-devo investiga la dimensión funcional de la evolución morfológica: (1) la relación entre la actividad y la adaptación de las partes, (2) la adaptabilidad ligada a la plasticidad ontogenética, y (3) la integración funcional de las estructuras orgánicas y sus consecuencias para la dinámica evolutiva. A la luz de los desarrollos de esta nueva 'evo-devo funcional', defenderemos que la distancia teórica entre la teoría sintética de la evolución y la evo-devo no puede interpretarse ya en términos de dos programas de investigación dirigidos a los distintos explananda de la función y de la forma, respectivamente. Desde nuestra perspectiva, la evo-devo de las funciones implica una radical reconceptuación de la noción de función biológica (como actividad y como organización) que, a su vez, plantea nuevas formas de síntesis entre la evo-devo y la genética de poblaciones.

\section{LA ACTIVIDAD DE LAS PARTES}

Cuando en la práctica biológica se atribuye una función a un rasgo, la noción de 'función' puede hacer referencia, o bien a 'lo que hace' (la función como actividad), o bien a aquello 'para lo que se usa' (el rol biológico o la función como uso) (Bock y von Wahlert 1965). La locomoción, por ejemplo, es la función de las extremidades de los vertebrados terrestres, que tiene asociados distintos roles, como huir de un predador o moverse hacia una fuente de comida. Partiendo de la célebre distinción de Mayr (1961) entre causas próximas y últimas, los filósofos de la biología suelen asociar ambos conceptos de función a ámbitos disciplinarios distintos: el 'rol biológico' a la biología evolucionista (pues el uso de un rasgo le confiere el valor adaptativo por el cual fue seleccionado), y la 'actividad' a la fisiología (Amundson y Lauder 1994, Wouters 2005, Love 2007).

Sin embargo, la importancia de la noción de actividad y su vinculación con el concepto de rol biológico en el contexto de la evo-devo demuestra que este dualismo epistemológico es incapaz de capturar la heterogeneidad semántica de la noción de función en la práctica biológica y, en particular, en la biología evolucionista (Wouters 2005).

Por un lado, en la tradición morfológica la investigación de las funciones ha estado siempre asociada a la caracterización estructural de las partes. En la morfología funcional, la adaptación de un rasgo nunca apela a éste comprendido en abstracto, sino que se refiere siempre a un rasgo cuya forma y estructura le confiere ciertas capacidades, las cuales, asociadas a ciertos roles y ejercidas con distinta eficacia, le permiten interactuar con la fuerza selectiva (Bock y von Wahlert 1965, Bock 1980, Bock 1988, Bock 1994). Por ejemplo, el rol biológico 
de la mandíbula vertebrada (el rol predatorio de cierta especie en un entorno ecológico) se examina en virtud de las capacidades biomecánicas asociadas a la forma mandibular. En este marco teórico, la evo-devo ha comenzado a investigar las consecuencias evolutivas que se derivan de las diferencias en la regulación ontogenética de la actividad y, por lo tanto, de la eficacia en el desempeño de sus funciones, de ciertos rasgos (Breuker et al. 2006). Continuando con nuestro ejemplo: ¿cómo los genes implicados en el desarrollo de la forma mandibular regulan la biomecánica del movimiento de la mandíbula?, ¿y cómo las diferencias individuales entre ellos dan lugar a diferencias adaptativas que pueden determinar la dinámica evolutiva? (Albertson et al. 2005)

Por otro lado, la biología del desarrollo ha revelado cómo el propio ejercicio de las actividades orgánicas puede determinar la forma de ciertas partes. Es el caso de la dependencia de la estructura de las venas de su uso por la circulación, la correlación entre la dieta y el desarrollo del intestino o el codesarrollo de la extremidad vertebrada y ciertas partes del sistema nervioso (Michel y Moore 1995, pp. 298-299). Las consecuencias teóricas de este tipo de adaptación fisiológica para la biología evolucionista vuelven a poner en evidencia el atomismo adaptacionista: la optimización de ciertas estructuras podría resultar, no de un proceso de 'perfeccionamiento' selectivo, sino de un proceso autónomo de adaptación.

Tanto en el estudio del efecto formativo de las actividades orgánicas como en la investigación de la variación del desarrollo asociada al rendimiento de las actividades orgánicas, la noción de actividad juega un rol central en la explicación de fenómenos evolutivos.

\section{AdAPTABILIDAD Y PLASTICIDAD ONTOGENÉTICA}

La plasticidad ontogenética es otra de las cuestiones que ha favorecido la irrupción del problema de la función en el programa de investigación de la evodevo. La llamada 'ecología del desarrollo' ha demostrado que las interacciones ambientales con los procesos ontogenéticos pueden ser responsables de cambios adaptativos tales como ciertas adaptaciones estacionales, cambios morfológicos que permiten escapar de los depredadores, la determinación de las castas en insectos dependiendo de la dieta o la especificación del sexo en función de la temperatura (Gilbert 2004).

La integración del estudio de la interacción de los sistemas ontogenéticos con el ambiente y sus consecuencias para la dinámica adaptativa ha dado lugar a la fundación de una nueva disciplina, la ecología evolutiva del desarrollo, que extiende más allá de las fronteras del organismo la agenda de la evo-devo (Gilbert y Epel 2008). Y es que si bien el carácter no hereditario de las 'adaptaciones directas' al ambiente las excluyó de la teoría evolutiva moderna, hoy se reconocen diversos modos en los que las modificaciones posibilitadas por la 
plasticidad del desarrollo pueden heredarse. A lo largo de varias generaciones, tales variaciones pueden estabilizarse mediante mecanismos de integración genética: a través de sus 'normas de reacción' adaptativas, el organismo puede alterar su fenotipo sin necesidad de crear variación de novo y posteriormente acumular cambios genéticos que estabilicen alternativas morfológicas latentes (West-Eberhard 2003). En este contexto teórico, los organismos se interpretan como sistemas funcionales cuya flexibilidad ante los cambios ambientales provee a la selección de un mayor espectro de fenotipos sobre los que actuar. Los sistemas de desarrollo facilitan, así, la evolución adaptativa, dando cuenta de la 'evolucionabilidad'. En este sentido, la evo-devo reconoce que la selección debería favorecer aquellos sistemas generativos que aumenten el potencial evolutivo de sus portadores, lo cual transforma radicalmente la propia conceptuación de la selección natural, que deja de ser, exclusivamente, un mecanismo productor de adaptaciones. Este nuevo nivel de selección, que «no actúa ni sobre el fenotipo ni sobre el genotipo, sino sobre las propiedades emergentes de los sistemas ontogenéticos» (Alberch 1991), se concibe como un proceso que, al promover la capacidad de evolucionar, selecciona mecanismos generadores de adaptabilidad (es decir, de variación no letal) que hacen que la propia evolución sea posible.

\section{LA ORGANIZACIÓN DE LAS TOTALIDADES ORGÁNICAS: MODULARIDAD E INTEGRACIÓN}

Los organismos son, a la vez, sistemas modulares (consisten en partes reconocibles porque están integradas internamente y son relativamente independientes de las otras) e integrados (las conexiones entre estos módulos no son simétricas, sino que unos partes están más conectados que otros). Si bien la evo-devo ha interpretado ambas dimensiones de la organización biológica desde una perspectiva esencialmente morfológica, ciertos autores han examinado la modularidad y la integración en un sentido funcional.

Si bien la modularidad suele interpretarse como resultado de la conectividad espacial de las partes, la individualidad de las partes se ha definido también en virtud de diferentes tipos de interacciones, como las correlaciones funcionales u ontogenéticas (Klingenberg 2004), y algunos autores han tratado de comprender cómo se relacionan los diferentes tipos de módulos. El hecho de que los módulos ontogenéticos sean autónomos en su desarrollo y de que los módulos funcionales sean también independientes de otros rasgos en sus efectos adaptativos permitiría una adaptación rápida y específica a condiciones ambientales cambiantes (Wagner 1989). En este sentido, en la evo-devo la construcción modular de los animales no sólo se concibe como un factum que ha de explicarse desde la perspectiva del desarrollo, sino que aparece también 
como uno de los principales factores de adaptabilidad que posibilitan el cambio evolutivo.

Al igual que la modularidad, la integración fenotípica se ha abordado tanto desde una perspectiva estructural como funcional. En el primer caso, con un espíritu similar al de la morfología teórica, uno de los grandes objetivos de la evo-devo ha consistido en desvelar las reglas ontogenéticas que permiten construir los grandes planes corporales. En el segundo, la integración fenotípica se ha interpretado en términos de subordinación funcional. En este contexto, la función se comprende como una «integración entre las partes tal que el todo 'funcione' en el sentido de mantener la integridad del organismo» (RasskinGutman 2003).

La anatomía comparada de Georges Cuvier fue el primer programa de investigación que se propuso investigar la diversidad morfológica a la luz de la idea kantiana de organización. ${ }^{2}$ En este marco teórico, la subordinación jerárquica de las partes animales revela que no todas ellas tienen el mismo valor, sino que su importancia depende precisamente de la función que desempeñan en la totalidad orgánica. Así, el 'principio de la subordinación de caracteres' explica que las estructuras a las cuales el resto está subordinado (como la médula espinal) sean las más constantes y su transformación implique la drástica transformación de las demás, mientras cuanto menos importantes son las partes, la variación se incrementa exponencialmente.

El concepto de función como organización que articula la perspectiva cuveriana de las condiciones de existencia es, por tanto, muy distinto, de la noción darwinista de adaptación ecológica (Etxeberria y Nuño de la Rosa 2009). La noción de organización, definida en el contexto de la anatomía fisiológica, es un concepto inmanente de función que, al dirigir la mirada a las dependencias internas que regulan las relaciones entre las partes animales, permite comprender las semejanzas entre las especies que comparten un mismo plan de organización. La noción de adaptación, sin embargo, se nutre de los interrogantes característicos de disciplinas muy distintas (la sistemática y la recién fundada biogeografía) que enfatizan las peculiaridades de los organismos y la forma en que las especies difieren en relación a sus entornos particulares y sus modos de vida. ${ }^{3}$

2 La noción de función como organización se remonta a la discusión kantiana del concepto de organismo, donde el concepto de función aparece asociado a una teleología inmanente que fundamenta los fines en las necesidades internas de la propia organización biológica: una totalidad es teleológica cuando sus partes están entrelazadas de modo que su dependencia recíproca está subordinada al cumplimiento de un fin común: el mantenimiento de la totalidad orgánica a la que pertenecen (Kant 1973, § 65).

3 En este sentido, y si bien Darwin sostiene en el Origen que la teoría de la selección natural permitía explicar la adaptación de los organismos a sus condiciones de existencia, en realidad este término había sido completamente redefinido. Como ha subrayado Caponi (2011), 
Las resistencias de Cuvier a aceptar la evolución de las especies, la focalización de la morfología evolucionista en el problema de la Forma, y la reducción sintética de la noción de función a la adaptación ecológica explican que el enfoque cuveriano de los planes de organización no se integrase en la biología evolucionista. Si bien autores como Schmalhausen continuaron recordando el rol jugado por la integración funcional en el proceso evolutivo, hasta los años ochenta no empezó a recuperarse la perspectiva organicista de los sistemas vivos en el marco de la morfología funcional (Arnold et al. 1989). No obstante, fue la teoría sistémica de Rupert Riedl (1978) la obra clave que permitió incorporar la integración funcional de las partes en la investigación del origen y la estabilidad evolutiva de la forma desde la perspectiva del desarrollo. Según el concepto de 'carga' (burden), la probabilidad de que un carácter evolucione depende del número e importancia de las funciones y caracteres que dependen de él (Wagner y Laubichler 2004). A lo largo de la evolución, la carga de un carácter puede aumentar, volviéndolo prácticamente invariable, como los caracteres de los planes corporales, o decrecer hasta el extremo de hacerlo desaparecer. La columna vertebral, por ejemplo, está altamente constreñida dada su importancia en conferir soporte y flexibilidad a los sistemas nervioso y vascular, así como al acoplamiento muscular; por su parte, el pelo humano está en la periferia del organismo, pues pocas estructuras dependen de él. Desde esta perspectiva, la pertenencia de un carácter a un plan corporal no está definida por las semejanzas entre las formas, sino que se trata de una propiedad sistémica que depende de su integración en la red cambiante de interdependencias ontogenéticas y funcionales (Wagner y Laubichler 2004; Wagner 1989).

En el marco de la evo-devo, e inspirándose en Riedl, Schwenk y Wagner han interpretado las constricciones en términos funcionales para dar cuenta de la estabilidad fenotípica (Schwenk y Wagner 2001, Wagner y Schwenk 2000). La integración conduce a 'configuraciones evolutivamente estables' (CEE), sistemas de caracteres que cuya interacción produce la 'función adecuada'. En este sentido, la estabilidad morfológica no depende ni de las propiedades variacionales del sistema ontogenético ni de la constancia del medio, sino de las constricciones funcionales que limitan el universo de variación fenotípica. Desde esta perspectiva, las CEE tenderán a resistir las perturbaciones ambientales si no exceden el rango tolerado por la función del sistema, mientras, en caso contrario, los caracteres pueden liberarse inaugurando canales evolutivos alternativos. El concepto de burden, en definitiva, da cuenta tanto de la estabilidad como de la 'evolucionabilidad' de la forma.

la correlación entre los perfiles orgánicos y las exigencias ambientales fue un corolario y no un explanandum ya establecido explicado por la selección natural. 
Todas las teorías que han investigado el rol de la integración funcional en la estabilidad de la forma implican una forma de selección natural muy distinta de la selección local característica del adaptacionismo: la selección estabilizadora interna, que ejercería su influencia en un amplio rango de entornos. Una de las limitaciones principales de la aproximación funcional a la evolución morfológica procede precisamente de este 'pesimismo' sobre lo morfológicamente posible, que, en el caso de la evo-devo, se traduce en la imposibilidad de concebir ciertas novedades evolutivas: ${ }^{4}$ frente a la selección ambiental, "motor de la mutabilidad", la selección interna se considera responsable de la estabilidad morfológica (Schwenk y Wagner 2001).

La recuperación de la noción cuveriana de organización ${ }^{5}$ y su aplicación a la comprensión de la dinámica evolutiva tiene dos grandes consecuencias de calado teórico:

En primer lugar, la inclusión de las demandas funcionales internas en la comprensión de la dinámica evolutiva revela una diferencia filosófica fundamental que ha enfrentado a las perspectivas morfológica y funcional de la diversidad orgánica desde el siglo XIX. Como subrayara Balan, la dialéctica entre las visiones teleológica y morfológica de la diversidad orgánica, cristalizada en el célebre debate que enfrentó a Cuvier y Geoffroy en 1830, radica en las distintas concepciones de la legalidad que gobierna el morfoespacio (Balan 1979, pp. 173-174): a la morfología pura le preocupan las 'condiciones de manifestación', es decir, aquellas condiciones puramente formales que permiten imaginar el universo de lo morfológicamente posible; la anatomía teleológica, apegada a lo empírico, describe las 'condiciones de existencia' que posibilitan a las formas orgánicas habitar el mundo. La anatomía cuveriana ignora, por tanto, la cuestión de una ciencia de las formas posibles y considera sólo las combinaciones actualmente existentes, es decir, las totalidades funcionalmente armónicas. Pues bien: en la evo-devo contemporánea encontramos una tensión semejante. Desde sus orígenes, la evo-devo ha aspirado a desvelar los mecanismos ontogenéticos que constriñen el universo de lo posible sobre el que operará la selección natural, una aproximación conceptualmente afín a la morfología pura decimonónica y la morfología teórica contemporánea. Al contrario, la perspectiva organicista en evo-devo, con un espíritu muy cuveriano, se empeña en comprender las formas que, de facto, son capaces de habitar el morfoespacio. Evocando a Leibniz, Gilbert (2000) lo plantea explícitamente en estos términos: si bien muchas

4 El escepticismo de Cuvier ante el evolucionismo, de hecho, estaba arraigado en razones conceptualmente análogas: el perfecto equilibrio revelado por el principio de la subordinación de caracteres impedía la evolución de la organización.

5 De hecho, ciertos integrantes de la evo-devo han comenzado a reivindicar explícitamente la figura de Cuvier (Reiss 2009) 
formas pueden existir desde la perspectiva del desarrollo, sólo aquellas que son mutuamente compatibles (es decir, aquellas que pueden integrarse en el organismo o causar un cambio compensatorio) existen de hecho.

Por otro lado, la noción de selección interna rompe con el dualismo entre el estructuralismo, centrado en las constricciones intraorgánicas, y el funcionalismo externalista característico de la Síntesis Moderna, dirigido a investigar la relación organismo-entorno. Desde la perspectiva de la evo-devo funcional, las constricciones y la selección no constituyen factores causales independientes, pues la selección opera en el fenotipo no sólo una vez se ha completado el desarrollo, sino a lo largo de toda su ontogenia (Schwenk y Wagner 2004); y no sólo porque los organismos deban sobrevivir también al entorno embrionario, sino también por la lógica interna que impone el 'milieu intérieur'.

$* * *$

Nuestro análisis de los distintos programas de investigación que se dedican a estudiar el desarrollo y la evolución de las funciones orgánicas nos permite concluir que la distancia teórica entre la Síntesis Moderna y la evo-devo no puede interpretarse en términos de dos programas de investigación dirigidos a los distintos explananda de la función y de la forma. La dialéctica entre forma y función se revela, al contrario, una tensión interna a la propia evo-devo: al igual que en la primera mitad del siglo XIX se enfrentaron la morfología pura y la anatomía fisiológica, la evo-devo contemporánea está dividida entre los practicantes de una evo-devo morfológica y la todavía minoritaria evo-devo funcional.

Esta nueva evo-devo funcional implica una radical reconceptuación de la noción de función biológica. Por un lado, la incorporación de la noción de actividad a la investigación del desarrollo y la evolución de la forma ha dado lugar a dos nociones de adaptación que la distinguen del concepto darwinista de adaptación ecológica: tanto la adaptación resultante de la plasticidad ontogenética como los efectos formativos del ejercicio de ciertas actividades biológicas en el desarrollo pueden dar lugar a adaptaciones evolutivas que no resultan de la dinámica reproductiva de los individuos que componen las poblaciones biológicas, sino de la dinámica interna de los propios organismos. Por otro lado, la incorporación de la noción de organización a la comprensión de la dinámica evolutiva tiene consecuencias de gran calado teórico. La investigación de las constricciones funcionales que limitan las combinaciones posibles de las partes introduce un enfoque internalista de las funciones que permite reformular la dualidad internalismo/externalismo. 


\section{Agradecimientos}

Este trabajo se ha llevado a cabo gracias a la financiación de un contrato post-doctoral del Konrad Lorenz Institute for Evolution and Cognition Research.

\section{REFERENCIAS BIBLIOGRÁFICAS}

ALBERCH, P., 1980: «Ontogenesis and Morphological Diversification». Integrative and Comparative Biology 20: 653-667. ,1991: «From genes to phenotype: dynamical systems and evolvability». Genetica 84: 5-11.

ALBERTSON, R. C., J. T. STREELMAN, T. D. KOCHER y P. C. YELICK, 2005: «Integration and Evolution of the Cichlid Myible: The Molecular Basis of Alternate Feeding Strategies». PNAS 102: 16287-16292.

AMUNDSON, R., 1994: «Two concepts of constraint: Adaptationism and the challenge from developmental biology». Philosophy of Science: 556-578. ,1998: «Typology Reconsidered: Two Doctrines on the History of Evolutionary Biology». Biology and Philosophy 13: 153-177.

AMUNDSON, R., y G.V. LAUDER, 1994: «Function without purpose». Biology and Philosophy 9: 443-469.

ARNOLD, S. J., P. ALBERCH, V. CSÁNYI, R. C. DAWKINS, S. B. EMERSON, B. FRITZSCH, T. J. HORDER, J. MAYNARD SMITH, M. J. STARCK, y E. S. VRBA, 1989: Complex Organismal Functions: Integration and Evolution in Vertebrates, New York: Wiley Chichester.

BALAN, B., 1979: L'Ordre et le temps: l'anatomie comparée et l'histoire des vivants au XIXe siècle. Paris: Vrin.

BOCK, W. J., 1980: «The Definition and Recognition of Biological Adaptation». American Zoologist 20: 217-227.

1988: «The Nature of Explanations in Morphology». American Zoologist 28: 205-215.

, 1994: «Concepts and methods in ecomorphology». Journal of Biosciences 19: 403-413

BOCK, W. J., y G. von WAHLERT, 1965: «Adaptation and the Form-Function Complex». Evolution 19: 269-299.

BREUKER, C. J., V. DEBAT, y C. P. KLINGENBERG, 2006: «Functional evo-devo». Trends in Ecology \& Evolution 21: 488-492.

CAPONI, G., 2011. La segunda agenda darwiniana: contribución preliminar a una historia del programa adaptacionista. México: Centro de Estudios Vicente Lombardo Toledano.

CARROLL, S.B., 2005: Endless forms most beautiful: The new science of evo devo and the making of the animal kingdom, New York: W. W. Norton \& Company. 
ETXEBERRIA, A., y L. NUÑO DE LA ROSA, 2009: «Partes y funciones en el desarrollo y la evolución. Hacia un darwinismo sistémico». H. DOPAZO y A. NAVARRO,(Eds.) Evolución y Adaptación: 150 años después del Origen de las Especies. Valencia: SESBE: 465-474.

GILBERT, S. F., 2000: Developmental biology $6^{\mathrm{a}}$ ed., Massachusetts: Sinauer Associates, Inc.

,2004: «Ecological Developmental Biology: Developmental Biology Meets the Real World 1». Russian Journal of Developmental Biology 35: 346-357.

GILBERT, S. F., y D. EPEL, 2008: Ecological developmental biology: integrating epigenetics, medicine and evolution, Sunderly, MA: Sinauer Associates Inc.

GOULD, S. J., y R. C. LEWONTIN, 1979: «The Spandrels of San Marco and the Panglossian Paradigm: A Critique of the Adaptationist Programme». Proceedings of the Royal Society of London 205: 581-598.

KANT, I., 1973: Crítica del juicio, Méjico D.F.: Porrúa.

KLINGENBERG, C. P., 2004: «Integration, Modules, and Development». M. PIGLIUCCI y K. A. PRESTON (Eds.) Phenotypic Integration: Studying the Ecology and Evolution of Complex Phenotypes. Oxford University Press: 213 227.

LOVE, A. C., 2007: «Functional homology and homology of function: Biological concepts and philosophical consequences». Biology and Philosophy 22: 691708.

LOVE, A. C., y R. A. Raff, 2003: «Knowing your ancestors: themes in the history of evo-devo». Evolution and Development 5: 327-330.

MAYR, E., 1961. «Cause and Effect in Biology: Kinds of causes, predictability, and teleology are viewed by a practicing biologist». Science 134: 1501-1506.

MICHEL, G.F., y C.L. MOORE, 1995: Developmental psychobiology. An Interdisciplinary Science. Cambridge: MIT Press.

NUÑO DE LA ROSA, L., 2012: El concepto de forma en la biología contemporánea. Examen filosófico. Tesis doctoral dirigida por José Luis González Recio y Jean Gayon. Madrid: Universidad Complutense de Madrid, Université Paris 1-Sorbonne.

RASSKIN-GUTMAN, D., 2003: «Modularity: Jumping Forms within Morphospace». W. CALLEBAUT y D. RASSKIN-GUTMAN (Eds.) Modularity: understying the development and evolution of natural complex systems. Boston, MA: MIT Press: 207-220.

REISS, J. O., 2009: Not by design: retiring Darwin's watchmaker. Berkeley/Los Angeles: University of California Press.

RIEDL, R., 1978. Order in living organisms: a systems analysis of evolution, Chichester/ New York: Wiley.

SALAZAR-CIUDAD, I., J. JERNVALL, y S. A. NEWMAN, 2003. «Mechanisms of pattern formation in development and evolution». Development 130: 2027-2037.

SCHWENK, K., y G. P. WAGNER, 2001. «Function and the Evolution of Phenotypic Stability: Connecting Pattern to Process». American Zoologist 41: 552-563.

SCHWENK, K., y G. P. WAGNER, 2004. «The Relativisim of Constraints on Phenotypic Evolution». M. PIGLIUCCI y K. A. PRESTON (Eds.) Phenotypic Integration: 
Studying the Ecology and Evolution of Complex Phenotypes. Oxford University Press: 390-408.

WAGNER, G. P., 1989. «The biological homology concept». Annual Review of Ecology and Systematics 20: 51-69.

WAGNER, G. P., y M. D. LAUBICHLER, 2004. «Rupert Riedl and the Re-Synthesis of Evolutionary and Developmental Biology: Body Plans and Evolvability». Journal of Experimental Zoology (Mol Dev Evol) 302: 92-102.

WAGNER, G. P., y K. SCHWENK, 2000. «Evolutionarily stable configurations: functional integration and the evolution of phenotypic stability». Evolutionary biology 31: 155-218.

WEST-EBERHARD, M. J., 2003. Developmental plasticity and evolution, USA: Oxford University Press.

WOUTERS, A., 2005. «The Function Debate in Philosophy». Acta Biotheor 53: $123-$ 151.

LaURa NUÑo de la Rosa es investigadora postdoctoral en el Konrad Lorenz Institute for Evolution \& Cognition Research, Adolf Lorenz Gasse 2. A-3422 Altenberg, Austria.

Línea de investigación:

Filosofía e historia de la biología, en particular de la morfología, la biología del desarrollo y la evo-devo.

Publicaciones recientes:

NUÑO DE LA ROSA, L. 2010. «Becoming organisms. The development of organisation and the organisation of development». History and philosophy of life sciences 32: 289-316.

NUÑO DE LA ROSA, L., y A. ETXEBERRIA. 2010. «¿Fue Darwin el "Newton de la brizna de hierba"? La herencia de Kant en la teoría darwinista de la evolución». Endoxa (24): 185-216.

Dirección electrónica: laura.nuno@kli.ac.at 